\title{
Leiomiomatosis intravascular gigante con extensión a cavidades cardíacas
}

\author{
Catalina Hernández-Flórez*, Ruby I. Jojoa-Jojoa, Ángel A. García-Peña \\ y Wilson R. Bohórquez-Rodríguez
}

Departamento de Medicina Interna. Hospital Universitario San Ignacio. Pontificia Universidad Javeriana, Bogotá, Colombia

Recibido el 4 de enero de 2017; aceptado el 11 de agosto de 2017

Disponible en Internet el 7 de mayo de 2018

\section{PALABRAS CLAVE \\ Ecocardiografía; \\ Tumores; \\ Diagnóstico}

\begin{abstract}
Resumen Se presenta el caso de inusual crecimiento intracardíaco de leiomiomatosis intravascular gigante en una mujer de 38 años, en quien durante evaluación prequirúrgica de cirugía abdominal se documentó de manera incidental una masa en cavidades cardiacas derechas. Dada su presentación infrecuente y las dificultades en el abordaje diagnóstico y terapéutico, se realiza posterior revisión de la literatura.

(c) 2018 Sociedad Colombiana de Cardiología y Cirugía Cardiovascular. Publicado por Elsevier España, S.L.U. Este es un artículo Open Access bajo la licencia CC BY-NC-ND (http:// creativecommons.org/licenses/by-nc-nd/4.0/).
\end{abstract}

\section{Giant intravascular leiomyomatosis with extension to the cardiac cavities}

Abstract The case is presented of an unusual intracardiac growth of a giant intravascular leiomyomatosis in a 38 year-old woman. During a pre-surgical evaluation for abdominal surgery, the incidental finding of a mass in the right cardiac cavities was noted. Given its uncommon presentation and the difficulties in the diagnostic and therapeutic approach, a subsequent review of the literature was performed.

(c) 2018 Sociedad Colombiana de Cardiología y Cirugía Cardiovascular. Published by Elsevier España, S.L.U. This is an open access article under the CC BY-NC-ND license (http:// creativecommons.org/licenses/by-nc-nd/4.0/).

\section{Caso}

* Autor para correspondencia.

Correo electrónico: chernandezf@husi.org.co

(C. Hernández-Flórez).
Paciente de género femenino, de 38 años de edad, con antecedente de lupus eritematoso sistémico, nefropatía lúpica clase II, vasculitis en sistema nervioso central, leucemia monocítica crónica y hemorragia uterina anormal asociada a 


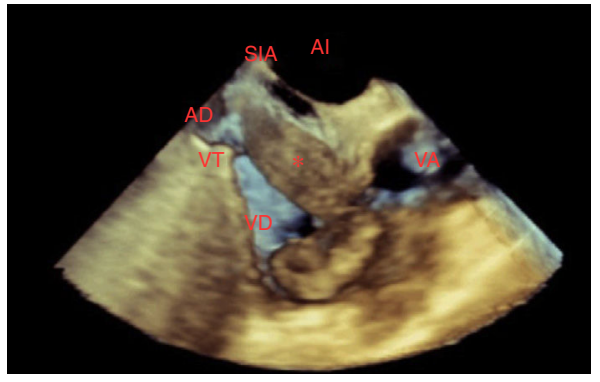

Figura 1 Proyección esófago superior $45^{\circ}$ modificada para enfocar la lesión $\left(^{*}\right)$. AD: aurícula derecha, VT: válvula tricuspídea, VD: ventrículo derecho, VA: válvula aórtica, Al: aurícula izquierda, SIA: septum interauricular.

miomatosis uterina, quien ingresó remitida a urgencias por hallazgos anormales en ecocardiograma transtorácico realizado durante evaluación prequirúrgica de histerectomía por miomatosis diagnosticada un año atrás. El ecocardiograma transtorácico extrainstitucional reportó masa versus trombo en septum interauricular proyectado hacia ventrículo derecho con dimensiones de $2,7 \mathrm{~cm}$ por $1,5 \mathrm{~cm}$, sin compromiso hemodinámico, crecimiento de cavidades cardiacas o valvulopatías relevantes con función biventricular preservada. Al ingreso negó sintomatología aguda. En revisión por sistemas se documentó disnea y clase funcional II/IV de tres años de evolución, sin deterioro en los últimos meses. Al examen físico se observó paciente en buenas condiciones, signos vitales y auscultación cardiopulmonar normales con leve dolor en hipogastrio a la palpación profunda y útero palpable de ubicación suprapúbica. Los estudios paraclínicos iniciales descartaron actividad lúpica; la química sanguínea estuvo dentro de límites normales, por lo que se le practicó ecocardiograma transesofágico que mostró como datos relevantes leve dilatación de la aurícula derecha con presencia de imagen hiperecogénica en forma de $L$, con ecodensidad de tejidos blandos, bordes regulares, aspecto lumbricoide, ubicada principalmente en la parte superior de la aurícula derecha con prolapso hacia ventrículo derecho, con diámetros intracavitarios de $65 \mathrm{~mm}, 34 \mathrm{~mm}$ con pedículo de $13 \mathrm{~mm}$ x $17 \mathrm{~mm}$, altamente móvil; sin embargo, no se observó punto de contacto o anclaje en estructuras cardiacas, con origen y llegada por la cava inferior, considerando como primera posibilidad la presencia de trombo versus lesión neoplásica (fig. 1). Se inició anticoagulación plena y se complementaron estudios con cavografía, que reportó extenso defecto de repleción en el eje ilíaco izquierdo, compromiso desde vena hipogástrica, vena cava inferior en toda su extensión, compromiso aproximadamente del $70 \%$ del calibre del vaso y trombo desde el eje de la ilíaca hasta la aurícula derecha. Se decidió realizar angiotomografía torácica, abdominal y pélvica con fase arterial y venosa, en la que se observó masa multilobulada dependiente de fondo uterino, con densidad heterogénea y realce periférico, con dimensiones de $14 \mathrm{x}$ $8 \times 13 \mathrm{~mm}(\mathrm{~L} \times \mathrm{AP} \times \mathrm{T})$, que mostró aporte arterial y drenaje venoso de sistemas ilíacos internos de forma bilateral; además de defecto de llenado central que iniciaba desde la aurícula derecha, comprometiendo las venas cava inferior en toda su extensión, renal izquierda, ilíaca común e hipogástrica izquierda; en la fase arterial mostró realce con medio de contraste e imagen que sugirió extensión de mioma uterino a vena ilíaca interna izquierda que se continuó con imagen previamente descrita, considerándose diagnóstico de leiomiomatosis intravascular (fig. 2). En junta médica se decidió efectuar extracción quirúrgica mediante histerectomía subtotal y extracción de toda la masa endovascular localizada en las venas ilíaca externa e interna, con longitud aproximada de $30 \mathrm{~cm}$ y $2 \mathrm{~cm}$ de ancho (fig. 3). Se comprobó por ecocardiograma transesofágico intraoperatorio la extracción completa de la masa. El reporte de Patología de la masa uterina confirmó el diagnóstico de leiomiomas celulares submucosos y subserosos con cambios degenerativos, mientras que la lesión intravascular corroboró la naturaleza tumoral con reporte de leiomioma celular vascular. La paciente tuvo evolución postoperatoria adecuada y permanece en seguimiento multidisciplinario ambulatorio.

\section{Discusión}

La leiomiomatosis es una enfermedad común en las mujeres, que en algunas ocasiones ocurre con patrones de crecimiento inusuales ${ }^{1}$. Puede confundirse con enfermedades malignas y llevar por tanto a errores diagnósticos y terapéuticos. Algunos ejemplos son la leiomiomatosis peritoneal difusa y los leiomiomas benignos metastásicos, retroperitoneales e intravasculares.

La leiomiomatosis intravascular es una complicación rara. Se trata de una extensión intravascular desde un leiomioma o desde la pared de un vaso uterino, la mayoría de veces a través de la vena ilíaca hasta la vena cava inferior $^{2}$. Es una condición que ocurre más comúnmente en mujeres premenopáusicas ${ }^{3}$, cuya aparición ocurre durante el período de actividad reproductiva, luego de lo cual se estabilizan o involucionan después de la menopausia; adicionalmente, pueden aumentar de tamaño y desarrollar atipia durante el embarazo o durante la administración de anticonceptivos orales. Hay dos teorías acerca del origen de la leiomiomatosis intravenosa: pueden surgir de la pared de las venas dentro del miometrio o ser el resultado de una invasión vascular extensa inusual a partir de leiomioma uterino ${ }^{4}$. El compromiso cardiaco (leimomatosis intracardiaca) se puede ver hasta entre el 10 y $40 \%$ de los $\operatorname{casos}^{1,5}$. Siempre es de crecimiento lento, por lo cual se espera no existan síntomas cardiovasculares hasta su extensión a nivel intracardiaco donde usualmente se presentan síntomas asociados a obstrucción del flujo salida del ventrículo derecho como síncope $e^{2,6,7}$ disnea, taquicardia ${ }^{8}$ y falla cardiaca 9 . Llama la atención, sin embargo, que en el caso reportado la paciente no refería síntomas agudos de origen cardiovascular y que el hallazgo de la masa cardiaca fuera incidental; en la revisión por sistemas únicamente se documentó disnea con clase funcional de II/IV de 3 años de evolución sin poder determinar si esta era secundaria a leiomiomatosis intracardiaca. Se han reportado casos con ausencia de síntomas ${ }^{10,11}$. En una serie de casos de 194 pacientes presentado por Li et al. ${ }^{12}$, se describen hasta $13 \%$ de pacientes asintomáticos cardiovasculares. La mayoría de las pacientes tiene historia de histerectomía por miomatosis uterina (53\%) o presentan miomas coexistentes en el momento del diagnóstico $(29 \%)^{12}$ como ocurrió con la paciente del caso. El diagnóstico generalmente se confirma mediante estudios ecocardiográficos tras los síntomas cardiovasculares e imágenes para eva- 

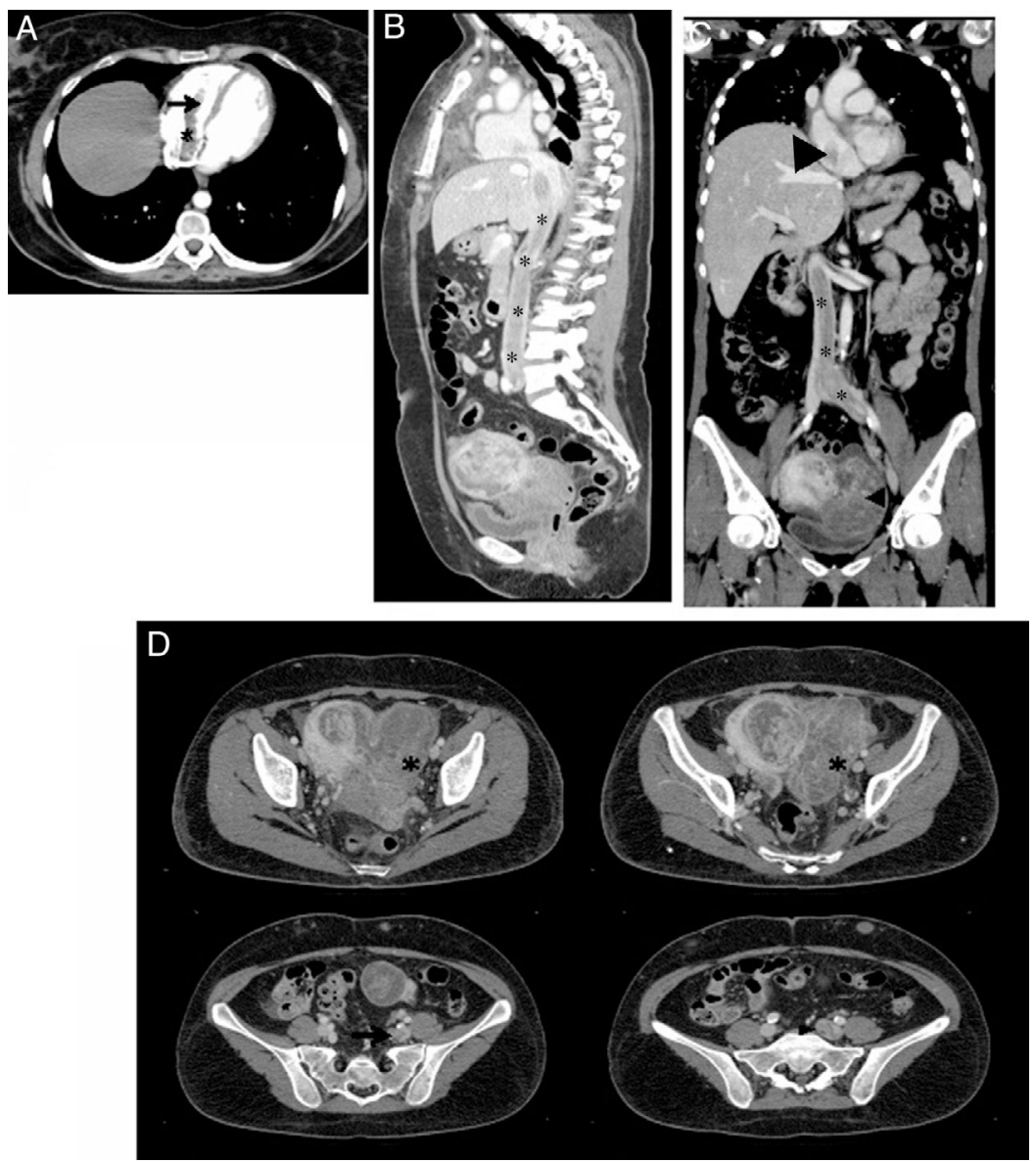

Figura 2 Tomografía de tórax y abdomen en fase venosa, A. Corte axial. Trombo que ocupa la aurícula (asterisco) y el ventrículo (flecha) derechos. B. Corte sagital. Masa que ocupa la vena cava inferior en toda su extensión (asteriscos) C. Corte coronal. Masa que ocupa la vena ilíaca común izquierda y la cava inferior (asteriscos), y que se extiende hasta la aurícula derecha (flecha). Se observa además una masa uterina que corresponde a mioma. D. Cortes axiales. Masa uterina que corresponde a mioma (asteriscos) y masa que ocupa y dilata las venas ilíaca interna (flecha) y común (cabeza de flecha) izquierdas.

luar trayectos venosos tanto por angiotomografía como por angiorresonancia. Los hallazgos en la ecocardiografía revelan una masa en cavidades derechas, y otras características como extensión desde la vena cava inferior; su naturaleza móvil sin adherirse a estructuras cardiacas con bordes bien demarcados, unidos a la historia de la paciente, son las pistas que pueden sugerir que se trate de una leiomiomatosis intracardíaca ${ }^{13}$.

Dado el hallazgo incidental del caso presentado y el contexto de autoinmunidad, el diagnóstico inicial fue un trombo intracavitario que se extendía desde la vena cava inferior ante lo cual se inició anticoagulación terapéutica. En el estudio presentado por $\mathrm{Li}$ et al. el diagnóstico preoperatorio fue correcto en $54,3 \%$ de los casos mientras que en $30 \%$ fue erróneamente diagnosticado como mixoma y en $3,8 \%$ como trombo intracavitario. Hasta el momento no se documenta ninguna asociación entre lupus eritematoso sistémico y leiomiomatosis intravascular, de manera que este es el primer caso reportado en el que se presentan estas entidades de manera concomitante. A pesar de la histología benigna de la leiomiomatosis intravenosa, esta puede ser maligna en su modo de progresión pues la posibilidad de extensión vascular conlleva riesgo de muerte súbita9,13,14, así

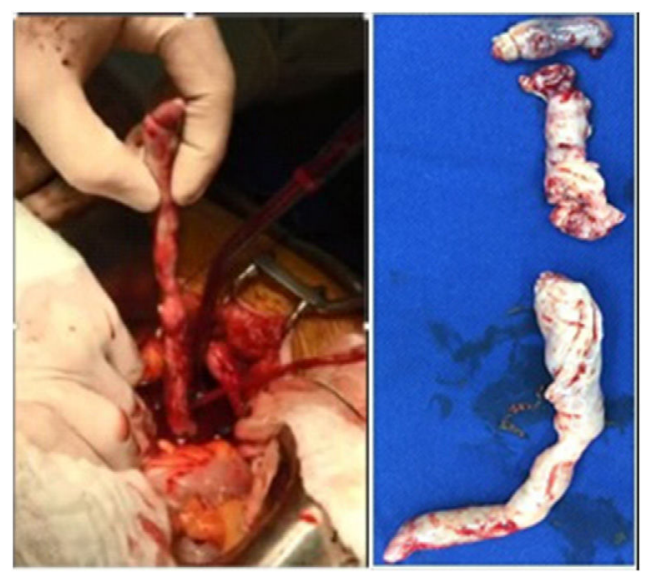

Figura 3 Pieza quirúrgica (fraccionada al momento de su escisión).

que se hace imperativo el tratamiento curativo con resección completa, que puede ser llevado a cabo con abordaje torácico y pélvico, en uno o dos momentos quirúrgicos ${ }^{11}$. La resección incompleta se asocia con recurrencia hasta en el 
$33 \%$ de las pacientes ${ }^{12}$. El tratamiento con bloqueo hormonal coadyuvante puede considerarse en los casos de resección quirúrgica incompleta; sin embargo, estudios retrospectivos sugieren que el uso de antiestrógenos como el tamoxifén no conlleva diferencia en la recurrencia de los $\operatorname{casos}^{12}$. Sólo se ha documentado por reporte de casos la participación de los inhibidores de aromatasa en la disminución de la recurrencia y progresión de la leiomiomatosis con crecimientos inusuales, incluida la leiomiomatosis intravascular ${ }^{15}$. En la literatura es claro que deberá ser estricto el seguimiento con imágenes en los casos en los que no se logre la resección completa, teniendo en cuenta el curso indolente de la enfermedad.

\section{Conclusión}

Aunque la leiomiomatosis intravascular intracardiaca es rara, puede ser más común de lo que se piensa. Su crecimiento lento y la posibilidad de presentación asintomática, hacen que el diagnóstico sea tardío y en ocasiones erróneo, características que dificultan su abordaje terapéutico adecuado. El tratamiento apropiado es la resección completa del leiomioma uterino y del tumor intravascular, que garantiza la curación sin recidiva y las bajas tasas de mortalidad; la extracción parcial puede producir recurrencia en un tercio de los casos. Su conocimiento por parte del gremio médico, es crucial para mejorar el pronóstico de estas pacientes.

\section{Financiación}

Ninguna.

\section{Conflictos de Interés}

Ninguno.

\section{Agradecimientos}

Al departamento de Cirugía cardiovascular y radiología del Hospital Universitario San Ignacio.

\section{Bibliografía}

1. Fasih N, Prasad A, Macnodald D, Fraser-Hill M, Papadatos D, Kielar A, et al. Leiomyomas beyond the uterus: unusual locations, rare manifestations. Radiographics. 2008;28:1931-48.

2. Sogabe M, Kawahito K, Aizawa K, Sato H, Misawa Y. Uterine intravenous leiomyomatosis with right ventricular extension. Ann Thorac Cardiovasc Surg. 2014;Suppl:933-6.

3. Price JD, Anagnostopoulos C, Benvenisty A, Kothuru RK, Balaram SK. Intracardiac extension of intravenous leiomyomatosis. Ann Thorac Surg. 2017;103:e145-7.

4. Tierney W, Ehrlich CE, Bailey JC, King RD, Roth LM, Wann LS. Intravenous leiomyomatosis of the uterus with extension into the heart. Am J Med. 1980;69:471-5.

5. Andrade LA, Torresan RZ, Sales JF, Vicentini R, De Souza GA. Case report intravenous leiomyomatosis of the uterus a report of three cases. Pathol Oncol Res. 1998;4:44-7.

6. Lee PK, David TE, Sloggett C, Ross JR. Intravenous leiomyomatosis with intracardiac extension: An unusual cause of cardiac syncope. Can Med Assoc J. 1990;142:1257-9.

7. Fornaris RJ, Rivera M, Jiménez L, Maldonado J. Multimodality evaluation of intravenous leiomyomatosis: a rare, benign but potentially life-threatening tumor. Am J Case Rep. 2015;16:794-800.

8. Stoleriu C, Rizas K, Gawaz M, Geisler T. Intracaval and intracardiac leiomyomatosis of uterine origin. Br Med J Case Reports. 2013;1:1-3.

9. Vega AF, Ortiz F, Morales A. Leiomiomatosis intravenosa. Rev Medica Sanitas. 2013;16:134-41.

10. Han MS, Choi IJ, Cha MS. A case of huge intravenous leiomyomatosis with intracardiac extension. J Women's Med. 2010;3:29-31.

11. Alizade K, Maddah G, Hosein Jafarian A, Khamene A, Jafarzadeh E, Mirzaeian S. Management of intravenous leiomyomatosis of uterous with extension to heart. Arch Iran Med. 2016;19:147-9.

12. Li B, Chen X, Chu YD, Li RY, Li WD, Ni YM. Intracardiac leiomyomatosis: A comprehensive analysis of 194 cases. Interact Cardiovasc Thorac Surg. 2013;17:132-9.

13. Li R, Shen Y, Sun Y, Zhang C, Yang Y, Yang J, et al. Intravenous leiomyomatosis with intracardiac extension_ Echocardiographic study and literature review. Texas Heart Inst J. 2014;41:502-6.

14. Shida $T$, Yoshimura $M$, Chihara $H$, Nakamura $K$. Intravenous leiomyomatosis of the pelvis with reextension into the heart. Ann Thorac Surg. 1986;42:104-6.

15. Doyle MP, Li A, Villanueva CI, Peeceeyen SCS, Cooper MG, Hanel $\mathrm{KC}$, et al. Treatment of intravenous leiomyomatosis with cardiac extension following incomplete resection. Int J Vasc Med. 2015:1-10. 